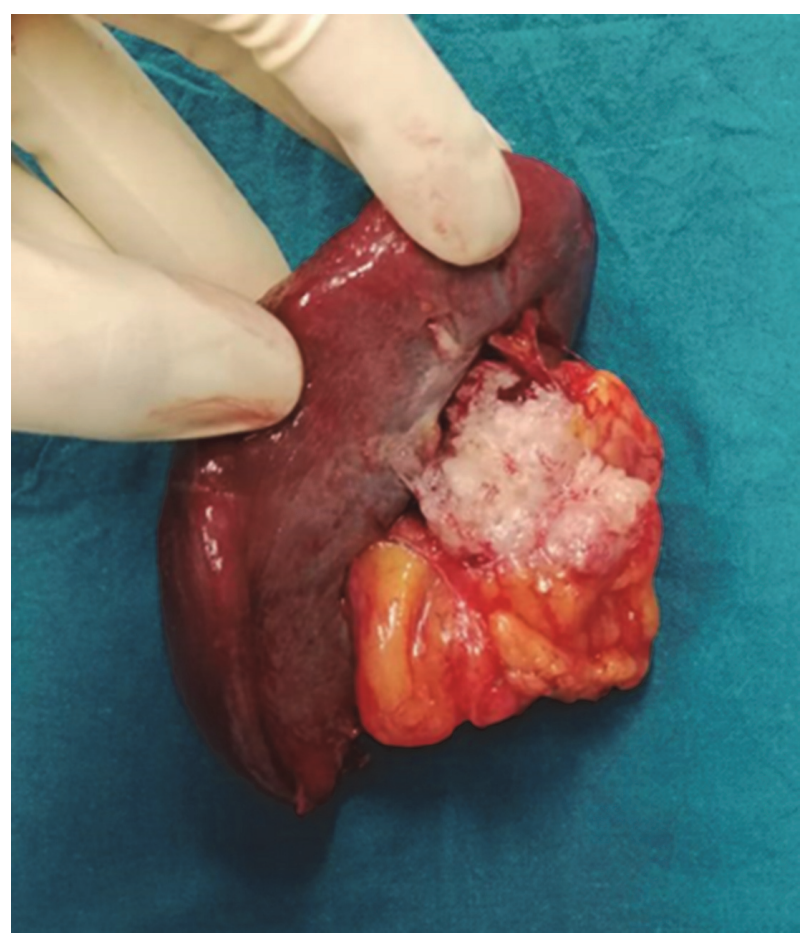

Abstract 605 Figure 1

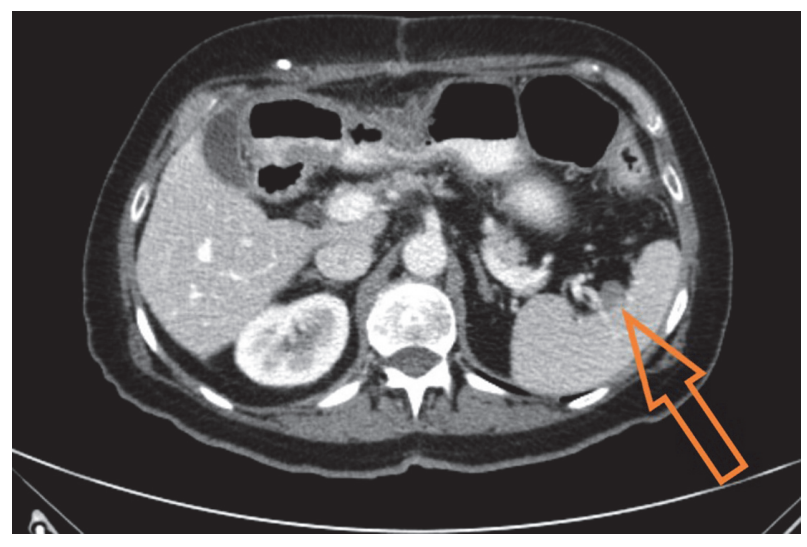

Abstract 605 Figure 2

follow-up time was 30.4 months. There were no differences in progression free-survival (long-rank $=0.069$ ) or overall survival (long-rank $=0.47$ ) between the groups.

Conclusion* Splenectomy in the course of debulking surgery for ovarian cancer does not seem to be associated with a higher rate of postoperative complications. Additionally, splenectomy does not have a deleterious influence before or during chemotherapy administration or a negative impact on oncological outcome.

\section{THE INCIDENCE OF ENDOMETRIOSIS IN PATIENTS WITH OVARIAN CANCERS}

A Ganovska*, S Kovachev. Military Medical Academy, Gynecology and Oncology, sofia, Bulgaria

10.1136/ijgc-2021-ESG0.438
Introduction/Background* Endometriosis is one of the most common gynaecological disorders. It affects $10-15 \%$ of all women in the reproductive years. Although endometriosis is recognised as a benign disease, its association with ovarian cancer has been frequently described in the medical literature since 1925 . The aim of our study was to determinate the incidence of endometriosis in patrients with proven ovarian cancer.

Methodology The study is retrospective, single-center and was conducted at the Clinic of General and Oncological Gynecology, Military Medical Academy-Sofia for a period of 2 years (2018-2020). The information from the history of the disease, operative protocol and histopathological examination were used. Included are 54 patients with histologically verified ovarian cancer operated at the Clinic of General and Oncological Gynecology. Preoperative tumor markers were examined in all patients. The staging of the disease is according to the FIGO classification. Histopathological preparations for the presence of endometriosis were revised in all patients.

Result(s)* The mean age of the patients enrolled in the study was 60.5 years (39 to 83 years). Depending on the histological type of ovarian cancer, the distribution is as follows: serous - 38 (70.4\%), mucinous - 6 (11.1\%), endometrioid - 2 (3.7\%), clear cell - 2 (3.7\%), granulosa cell - $3(5.6 \%)$.), small cell - 1 (1.8\%), seromucinous - 2 (3.7\%). Histologically, endometriosis was detected in $11(20 \%)$ of all patients. In patients with endometriosis, the most common histological type of ovarian cancer is serous - 6 (54.5\%).

Conclusion* According to our results, the incidence of endometriosis accompanying ovarian cancer is relatively high. Additional research is needed to look at the relationship between endometriosis as a precursor to ovarian cancer.

\section{GENETIC PROFILE BY WHOLE EXOME SEQUENCING OF BORDERLINE OVARIAN TUMORS: SERIES OF 32 PATIENTS}

${ }^{1} \mathrm{D}$ Atallah*, ${ }^{2} \mathrm{El}$ Feghaly, ${ }^{3} \mathrm{E}$ Choueiry, ${ }^{4} \mathrm{~N}$ Jalkh, ${ }^{5} \mathrm{~A}$ Khaddage, ${ }^{6} \mathrm{M}$ Akiki, ${ }^{7} \mathrm{H}$ Kourie, ${ }^{1} \mathrm{~N}$ El Kassis, ${ }^{7} \mathrm{G}$ Chahine, ${ }^{1} \mathrm{M}$ Moubarak. ${ }^{1}$ Hôtel-Dieu de France University Hospital, Saint Joseph University, Obstetrics and Gynecology, Beirut, Lebanon; ${ }^{2}$ Hôtel-Dieu de France, Saint Joseph University, Beirut, Lebanon; ${ }^{3}$ Lebanese American University, Human Genetics, Beirut, Lebanon; ${ }^{4}$ Saint Joseph University, Medical Genetics Unit, Beirut, Lebanon; ${ }^{5}$ HôtelDieu de France University Hospital, Saint Joseph University, Pathology, Beirut, Lebanon; ${ }^{6}$ Hôtel-Dieu de France University Hospital, Pathology, Beirut, Lebanon; ${ }^{7}$ Hôtel-Dieu de France University Hospital, Saint Joseph University, Oncology, Beirut, Lebanon

\subsection{6/ijgc-2021-ESG0.439}

Introduction/Background* Borderline ovarian tumors are defined as non-invasive epithelial ovarian tumors which can have an intraperitoneal extension. Molecular studies have shown a correlation between the patient's response to chemotherapeutic treatments adjunct to surgery and the tumor's genetic profile, especially related to the KRAS and BRAF genes. This study aims to assess the molecular profile of

BOTs in the Lebanese population by Whole Exome Sequencing (WES) and correlate the results with patients' clinical profiles.

Methodology 33 tumors belonging to 32 Lebanese patients presenting with BOTs, diagnosed at Hôtel Dieu de

France were included A total of 234 genes involved in different germinal and somatic types of cancer were analyzed using Next Generation Sequencing in the 33 included tumors. Genetic variants detected in more than $5 \%$ of the reads, with a sequencing depth $\geq 50 \mathrm{x}$, were selected. 